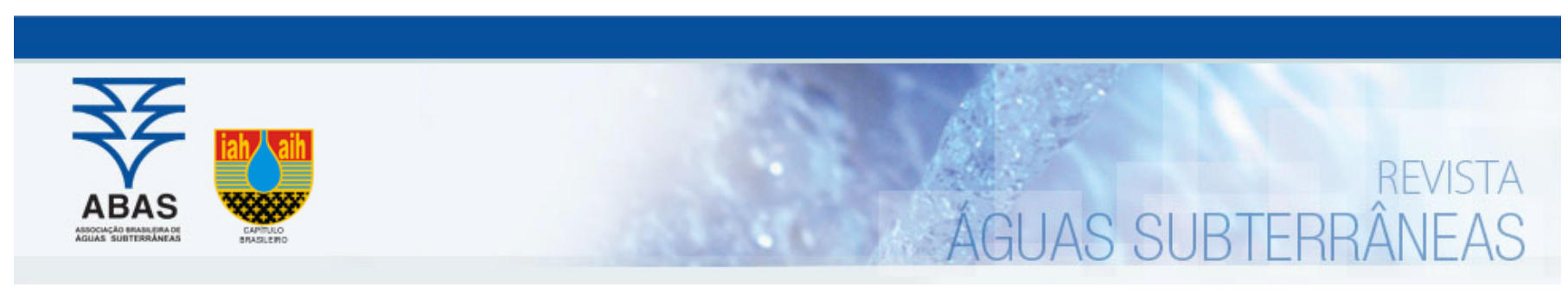

Artigos

\title{
As interações entre águas subterrâneas e superficiais em uma laguna costeira: Lagoa da Conceição, Florianópolis/SC, Brasil
}

\section{The groundwater-surface water interaction in a costal lagoon: Lagoa da Conceição, Florianópolis/SC, Brazil}

\author{
Thiago de Liz Arcari1; Priscilla Kern¹; Laura Sobral Verona2; Davide Franco1; \\ 1 Laboratorio de Hidraulica Maritima, Universidade Federal de Santa Catarina (LaHiMar/UFSC), Florianópolis, SC \\ 2 SiMCosta - Universidade Federal de Santa Catarina (UFSC), Florianópolis, SC
}

$凶$ thiagoarcari@gmail.com, priscilla.kern@gmail.com, laura.sverona@gmail.com, d.franco.ocean@gmail.com

\section{Palavras-chave: \\ Seepage meters. \\ Descargas de águas subterrâneas (DAS). Lagunas costeiras.}

Resumo

As interações entre as águas subterrâneas e superficiais (Asub-Asup) ocorrem em todos os tipos de corpos hídricos, como rios, lagos, lagoas costeiras e oceanos. A importância deste processo é cada vez mais reconhecida e estudada, onde recentes investigações no sul do Brasil identificaram grande importância das descargas de águas subterrâneas (DAS) nos aportes de nutrientes e ciclos biogeoquímicos do oceano. A área de estudo configura-se em uma laguna costeira sufocada, localizada na Ilha de Santa Catarina. 0 método de identificação e quantificação deste trabalho foi o seepage meter, que consiste em medições diretas e pontuais dos fluxos na interface sedimento-água. A metodologia apresentou resultados satisfatórios, identificando padrões de interação Asub-Asup em cinco estações amostrais. Os resultados apresentaram taxas de DAS variando de -12,0 a $6,0 \mathrm{~cm} / \mathrm{d}$, levando à proposta de modelos conceituais dos regimes de interação Asub-Asup localmente, onde presume-se que estes sejam afetados diretamente pelo uso dos recursos hídricos e variações nos gradientes hidráulicos.

Abstract

Keywords:

Seepage meters.

Submarine groundwater discharge (SGD).

Coastal lagoons.

Revisado por pares.

Recebido em: 29/08/2018.

Aprovado em: 07/12/2018.

The groundwater-surface water interaction (GW-SW) occurs in nearly all surface-water environments, such as rivers, lakes, lagoons and oceans. The reconnaissance of this process has grown rapidly. Recent studies in south Brazil have shown great influence of submarine groundwater discharge (SGD) in the Atlantic Ocean biogeochemical cycle. The study area is a choked coastal lagoon located in Santa Catarina Island, Brazil. In this study, seepage meters were used to identify and quantify GW-SW fluxes in the sediment-water interface. This methodology revealed satisfactory results of seepage fluxes patterns in five sampling stations. The results shown SGD rate from $-12,0$ to $6,0 \mathrm{~cm} / \mathrm{d}$. Conceptual modes were proposed for the GW-SW interaction in Lagoa da Conceição, which presumes that the use of water resources may directly affect seepage fluxes in the area.

DOI: http:/dx.doi.org/10.14295/ras.v33i1.29213

\section{INTRODUÇÃO}

As águas subterrâneas (Asub) e superficiais (Asup) foram historicamente estudadas e analisadas como recursos e compartimentos isolados. Estudos integrados da interface Asub-Asup vêm sendo desenvolvidos com o objetivo de quantificar balanços hídricos e avaliar possíveis efeitos das atividades humanas em rios, lagos, lagunas e oceanos (SOPHOCLEOUS, 2002).

O desenvolvimento de metodologias de quantificação dos fluxos de água entre estes compartimentos é um dos aspectos importantes dos estudos na interface Asub-Asup. Entre eles, destacam-se as metodologias diretas e pontuais (seepage meters, mini piezômetros e piezomanômetros), metodologias indiretas (uso de traçadores) e modelagem numérica integrada.
As interações e trocas entre as águas de superfície e as águas subterrâneas ocorrem em todos os tipos de corpos hídricos, como rios, lagos, lagoas costeiras e áreas alagadas, sendo essa interação dependente das características geológicas e climatológicas da região (WINTER et al., 1998).

A importância deste processo é cada vez mais reconhecida e estudada. Estudos recentes no sul do Brasil em lagoas costeiras apontaram fluxos importantes na interface sedimento-água e também identificaram grande relação entre as águas subterrâneas, os ciclos biogeoquímicos e o aporte de nutrientes (ANDRADE et al., 2012; ATTISANO, 2012; ATTISANO et al., 2008; BURNETT et al., 2006; DEBNATH; MUKHERJEE, 2016; Jl et al., 2013; LEOTE; IBÁNHEZ; ROCHA, 2008; NIENCHESKI et al., 2007; ROCHA; ANDRADE; NIENCHESKI, 2015; SANTOS et al., 2008). 
Em zonas costeiras, as Descargas de Águas Subterrâneas em sistemas marinhos (DAS, do inglês Submarine Groundwater Discharge) são definidas como todo e qualquer fluxo de água em margens continentais do sedimento para o oceano, independentemente da composição do fluido ou forças atuantes (BURNETT et al., 2003).

As DAS em ambientes costeiros foram por muitos anos negligenciadas cientificamente (BURNETT et al., 2003). Dentre as principais razões apontadas por Lewandowski et al. (2014) pode-se destacar o fato de que as DAS não serem visíveis e ocorrerem de forma difusa em uma grande área, fazendo com que as descargas locais sejam difíceis de mensurar. Além disso, as descargas são espacialmente e temporalmente heterogêneas, implicando em muitas medições de DAS para uma amostragem representativa. Apesar de existirem diferentes métodos para mensurar as DAS, ainda não existe um método simples, prático e eficaz.

Dentre os métodos existentes para a quantificação de DAS, o método de amostragem conhecido como seepage meter foi primeiramente descrito por Lee (1977). Este método consiste em cobrir e isolar uma pequena área da interface sedimento-água, enterrando a meia seção de um tambor com um coletor flexível acoplado, onde a água que flui entre a interface sedimento-água é então quantificada. Diversos estudos utilizaram esta metodologia, que se destaca por sua simplicidade, resposta rápida e baixos custos envolvidos, sendo uma metodologia importante para a investigação e fase confirmatória da quantificação das interações Asub-Asup em pontos específicos (ARANTES, 2003; BURNETT et al., 2006; DEBNATH; MUKHERJEE, 2016; LEOTE; IBÁNHEZ; ROCHA, 2008; MARTIN et al., 2007; ROCHA; ANDRADE; NIENCHESKI, 2015; SHAW; PREPAS, 1989; SOLDER et al., 2016).

A Lagoa da Conceição, localizada em Florianópolis/SC, é o quinto maior ambiente lagunar do Estado de Santa Catarina. Além da importância ecológica como local de abrigo e reprodução para diversas espécies de organismos marinhos, é um patrimônio paisagístico, arqueológico e econômico (PORTO FILHO, 1993; SIERRA DE LEDO; SORIANO-SIERRA, 1999). Nas últimas décadas, estudos mostram alterações das características hidroquímicas e biológicas desse corpo lagunar (CURY et al., 2017; FONSECA, 2006). Os processos que afetam as condições, variabilidade física e qualitativa da Lagoa da Conceição são de grande complexidade, por isso é necessária a utilização de uma abordagem integral deste sistema. Segundo Moore (2010), devido as águas subterrâneas apresentarem maiores concentrações de nutrientes, carbono e metais, as DAS são provavelmente mais importantes que as descargas fluviais para os ciclos biogeoquímicos dos oceanos e áreas costeiras.

Desta forma, este estudo objetiva desenvolver e utilizar o método seepage meter para a investigação e levantamento de dados primários da interação Asub-Asup na Lagoa da Conceição.

\section{MATERIAL E MÉTODOS}

\section{1. Área de Estudo}

A Lagoa da Conceição está localizada na região centro-leste da Ilha de Santa Catarina (6946807 N; 751632 L; 22 J) representada na Figura 1. A área total da bacia hidrográfica é de $82,1 \mathrm{~km}^{2}$.
Desta, a laguna ocupa uma área de $20,3 \mathrm{~km}^{2}$, estendendo-se por $13 \mathrm{~km}$ sentido norte-sul, orientado paralelamente à costa (BIER, 2013). A laguna é delimitada a oeste por encostas altas e íngremes do complexo cristalino, coberto por uma fina camada de argissolo densamente vegetado e a leste por depósitos de areias marinhas. Na parte sul, a laguna é separada do oceano por um grande campo de dunas (MUEHE; CARUSO JR., 1989).

A Lagoa da Conceição configura-se como uma laguna sufocada, com uma única conexão com o Oceano Atlântico, o Canal da Barra da Lagoa. O canal possui um total de $2,8 \mathrm{~km}$ de extensão e promove a troca de água entre a laguna e o oceano. Devido ao seu comprimento e meandros, o Canal da Barra da Lagoa atua reduzindo o efeito das marés no interior da laguna, funcionando como um filtro de alta frequência para as oscilações do nível d'água do oceano até a Lagoa da Conceição e atenua principalmente as componentes com período menor de 36h (maré astronômica) (ROCHA, 2007). Desta forma, o nível da laguna é afetado diretamente pela direção e magnitude dos campos de ventos regionais. Sistemas frontais do quadrante sul causam o empilhamento das águas do oceano, promovendo o fluxo do canal para dentro da laguna, levando ao aumento do nível d'água. Já a ocorrência de ventos do quadrante norte/nordeste proporcionam a saída de água da laguna e a consequente diminuição do nível d'água, como mostrou os estudos de Oliveira (2016), Odreski (2012) e Pereira (2004).

\subsection{Estações de Monitoramento}

Com o objetivo de identificar os diferentes padrões de interação Asub-Asup na Lagoa da Conceição, foram estabelecidas 5 estações de monitoramento (Figura 1). As estações foram definidas em função das diferentes características geomorfológicas da laguna e das sub-bacias adjacentes, sendo elas:

- Costa Cachoeira (CC): Estação localizada na porção oeste da laguna (6948726 N; 750398 L; 22J), próximo ao distrito da Costa da Lagoa. A sub-bacia hidrográfica adjacente se destaca pela alta declividade, formação geológica do complexo cristalino e densa vegetação.

- Costa Rio Vermelho (CRV): Localizada no estreito cordão lagunar-praial, na margem leste da laguna (6947527 N; $751925 \mathrm{~L}$; 22J). 0 cordão possui baixa declividade e elevação de poucos metros. É a estação mais próxima do oceano, sendo assim um local susceptível à interação de águas entre a laguna e o oceano.

- Ponta das Almas (PA): Localizada na margem oeste da laguna (6945174 N; 750734 L; 22J), apresenta características semeIhantes à estação CC, porém com maior urbanização e menor declividade da bacia adjacente.

- Rendeiras (RR): Estação localizada nas proximidades da Av. das Rendeiras (6943661 N; 751456 L; 22J). Esta estação está localizada próxima a um campo de dunas onde ocorre a infiltração artificial decorrente de uma lagoa de evapoinfiltração (LEI) de uma estação de tratamento de esgotos. Esta estação recebe o efluente de cerca de 15.000 habitantes.

- Lagoa late Clube (LIC): Localizada nas imediações da Lagoa late Clube (6943217 N; 749245 L; 22J). É um local de elevada urbanização, com a presença de poços de captação de águas subterrâneas para abastecimento público e presença de ponteiras para abastecimento domiciliar. 
Figura 1 - Localização da área de estudo, estações de monitoramento, sub-bacias adjacentes e eixos dos perfis praiais

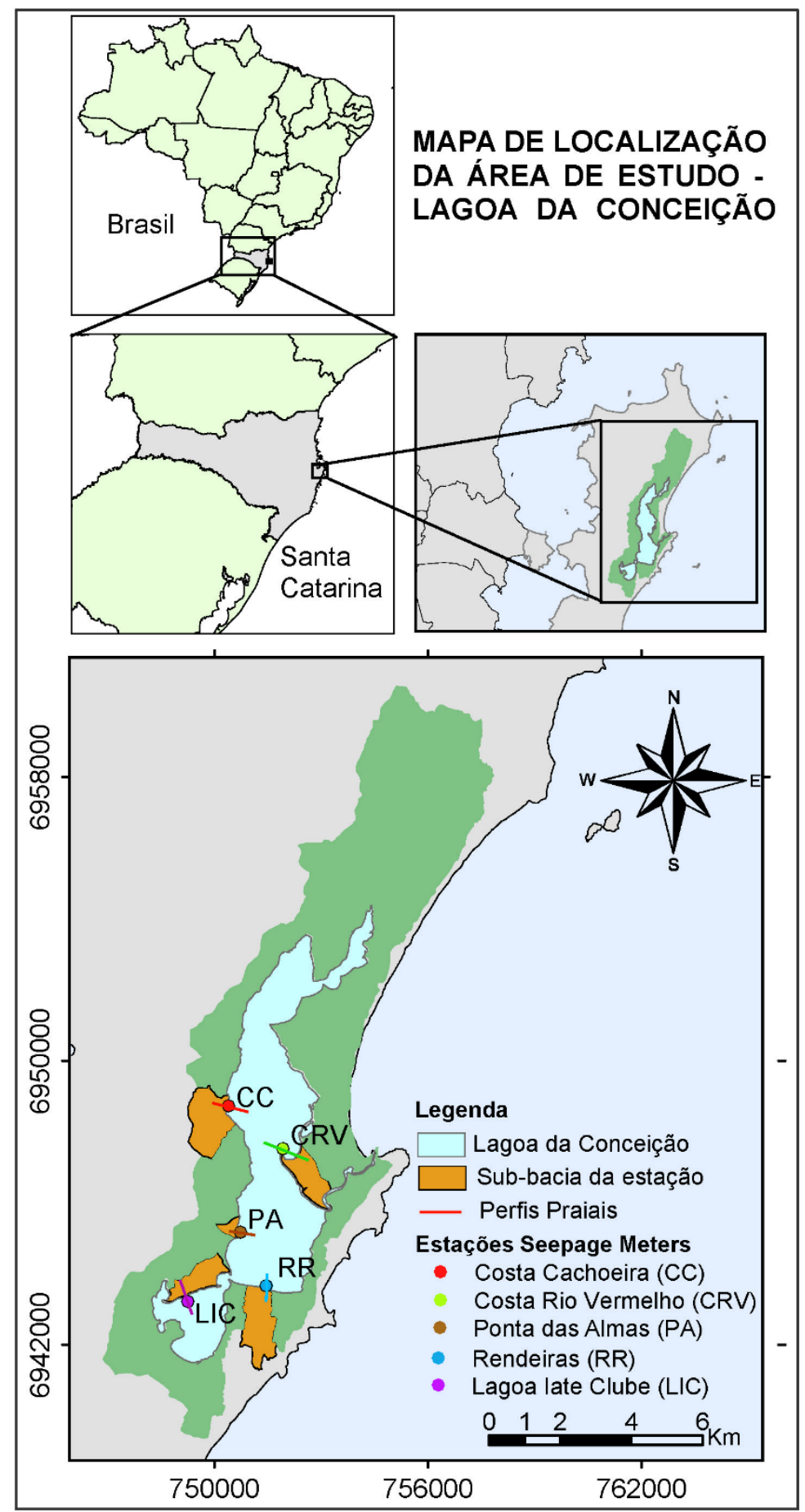

A Figura 2 apresenta os perfis praiais (topobatimétricos) dos transectos nas estações de monitoramento. A topografia foi obtida a partir do modelo digital de elevação, fornecido pela Secretaria de Desenvolvimento Sustentável de Santa Catarina. O levantamento batimétrico foi realizado no ano 2001 pela a empresa Aerolevan- tamentos e Consultoria S/A, a serviço do Instituto de Planejamento Urbano de Florianópolis (IPUF). Os perfis foram gerados a partir do acoplamento das duas bases de dados no software Global Mapper 17. 
Figura 2 -Perfis praiais e configuração dos transectos de seepage meters

Perfil Praial - Costa Cachoeira (CC)

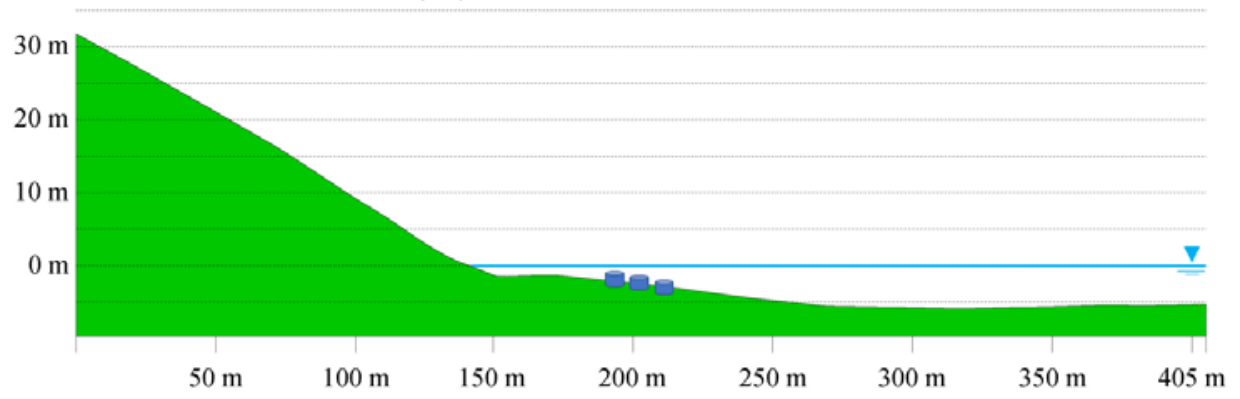

Perfil Praial - Costa Rio Vermelho (CRV)
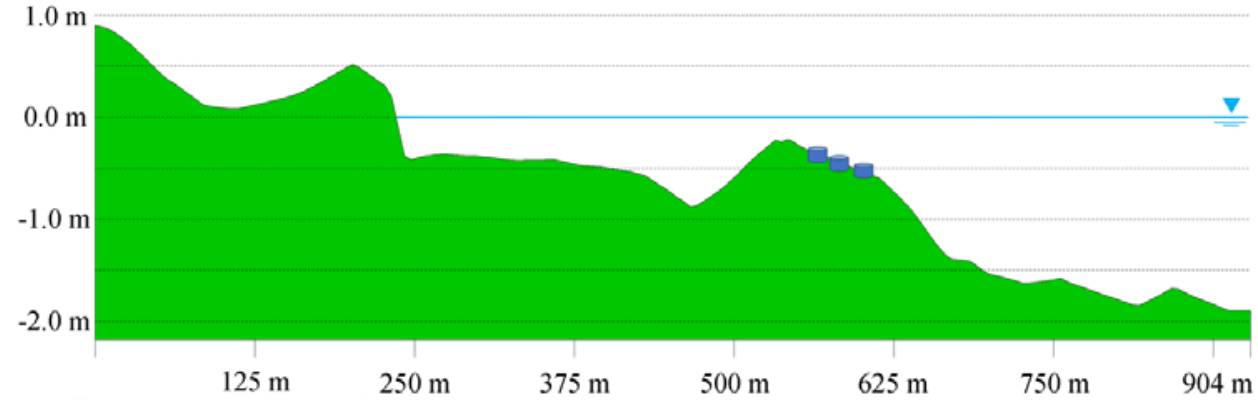

Perfil Praial - Estação Ponta das Almas (PA)

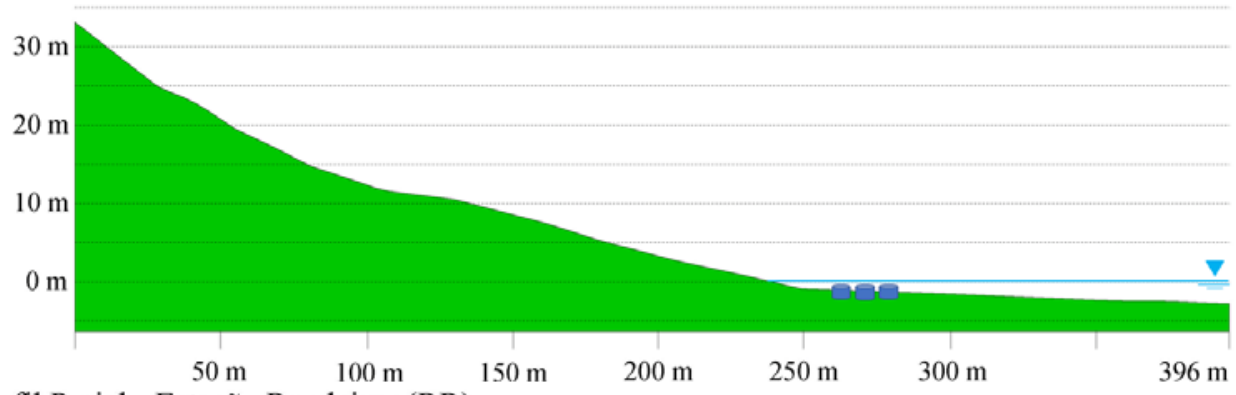

Perfil Praial - Estação Rendeiras (RR)

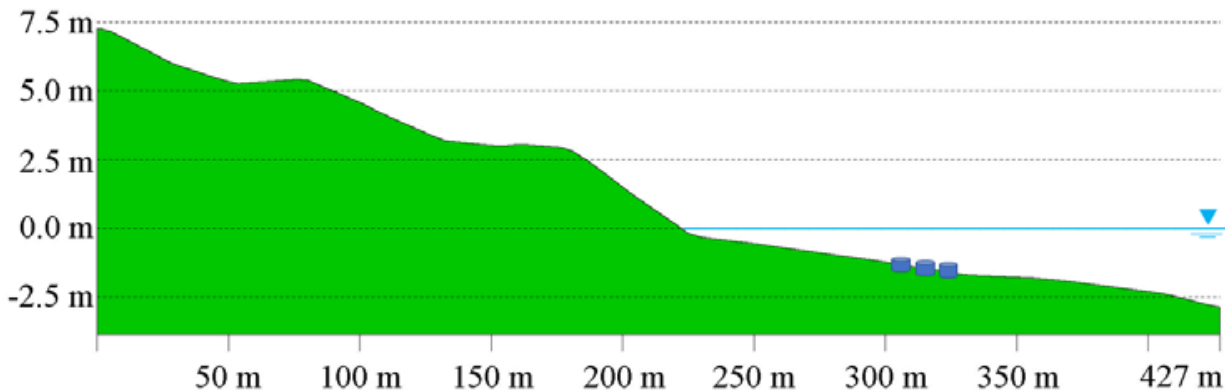

Perfil Praial - Estação Lagoa Iate Clube (LIC)

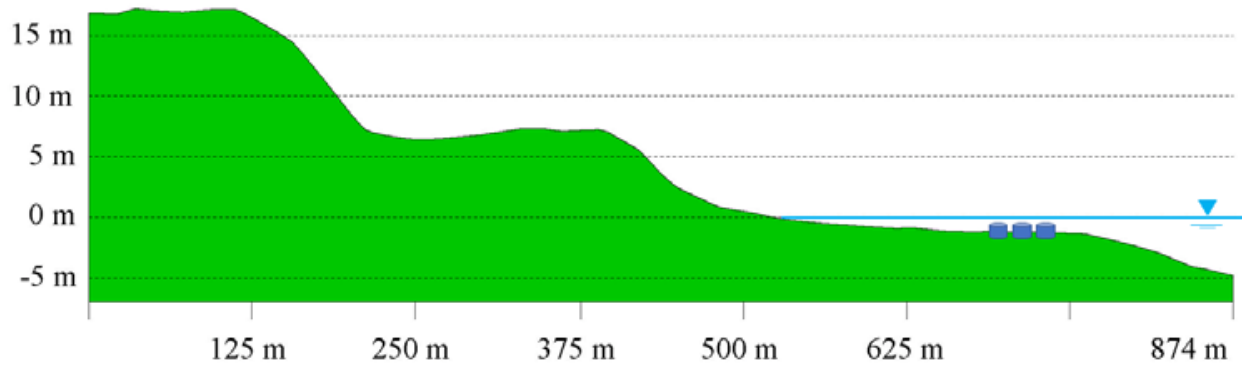

\subsection{Seepage Meter}

Para o levantamento de dados da interação Asub-Asup, foram confeccionados seepage meters, conforme metodologia descrita em Lee (1977). Foi convencionada a definição fluxo positivo para o movimento da água no sentido subterrâneo-superficial (descarga), enquanto que o fluxo contrário (infiltração) foi convencionado o termo fluxo negativo. A Figura 3 apresenta o esquema de funcionamento do equipamento. 
Figura 3 - Esquema de funcionamento do seepage meter e convenção dos sentidos do fluxo

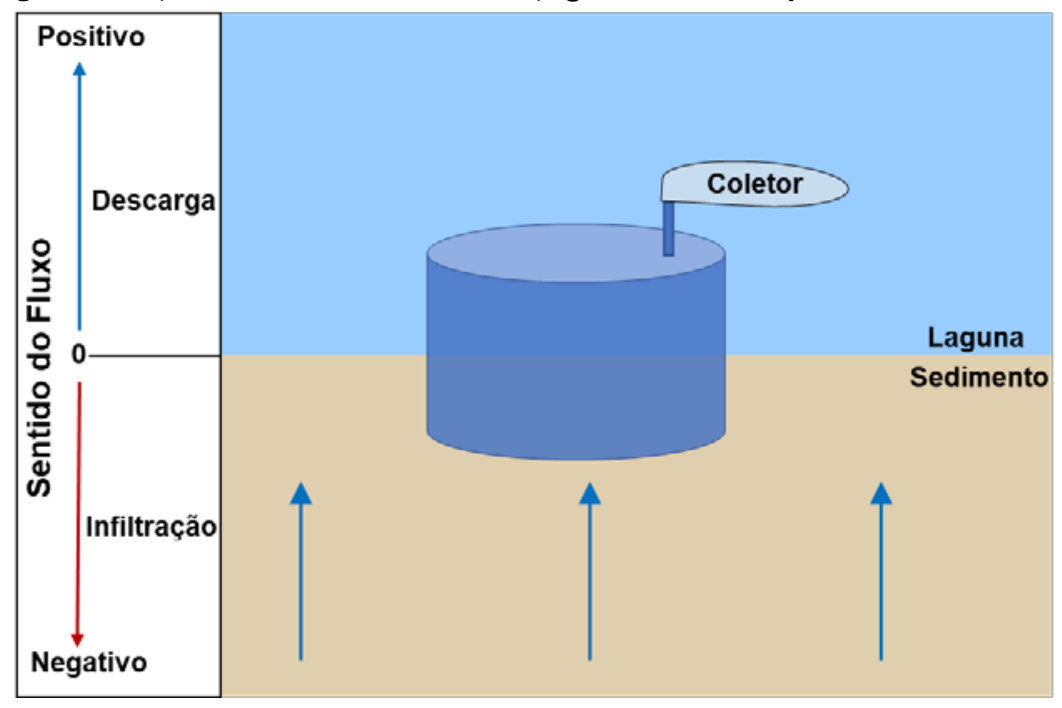

Foram realizados testes pilotos a fim de desenvolver o melhor design do equipamento para o uso na área de estudo específica. Tambores com maior diâmetro apresentaram resultados com menor variabilidade, como evidenciou o estudo de Rosenberry e Menheer (2006), que constatou que seepage meters com diâmetros maiores minimizavam os efeitos de heterogeneidade dos fluxos subterrâneos. 0 design utilizado neste estudo foi um tambor plástico de polietileno de alta densidade com volume de $200 \mathrm{~L}$ seccionado. 0 diâmetro interno dos tambores utilizados foi de 60 $\mathrm{cm}$ (Figura 4).

O coletor plástico acoplado ao tambor é a peça mais importante do equipamento e também a maior fonte de erros de medição (MURDOCH; KELLY, 2003). O coletor deve ter pequena espessura, ser flexível e maleável, de modo que cause a menor resistência possível ao fluxo d'água. Por outro lado, deve ser resistente o suficiente para não causar vazamentos. Após testes em campo, optou-se por um coletor plástico de polietileno de baixa densidade com espessura de 0,8 mm e dimensões 35 × $45 \mathrm{~cm}$ (Figura 4).

O coletor foi conectado ao tambor com o uso de um flange de 25 or com o uso de um flange de $25 \mathrm{~mm}$ com o-ring para vedação, registro de esfera de $25 \mathrm{~mm}$ e um tubo conector (Figura 3). A fixação do coletor plástico no tubo conector foi feita pela amarração com câmara de ar de pneu automotivo, garantindo a vedação completa. 0 registro tem a função de evitar perdas ou ganhos de água durante a inserção e retirada dos coletores nos seepage meters.

Figura 4 - Seepage meters, coletores plásticos e conexões

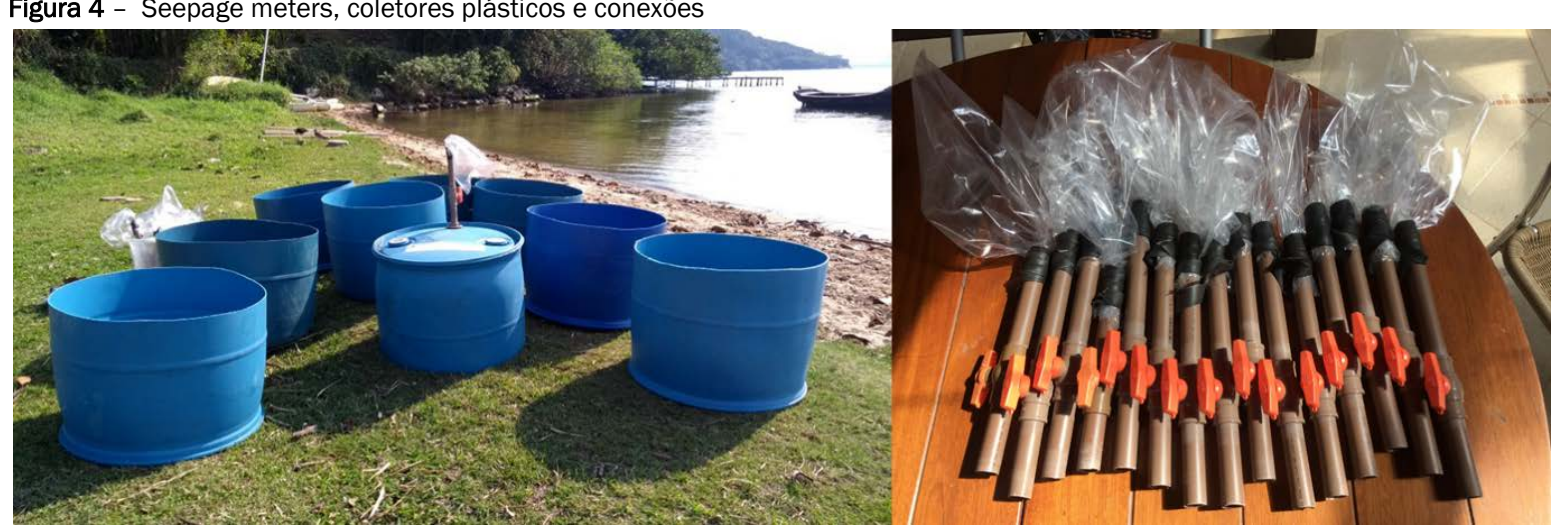

A instalação dos seepage meters no sedimento da laguna foi realizada em local livre de vegetação e pedras. Os equipamentos foram inseridos no sedimento de modo a atingir vedação adequada. No caso de areias finas e médias, uma inserção superior a $15 \mathrm{~cm}$ é considerada apropriada (SOLDER et al., 2016). Os equipamentos foram instalados com leve inclinação, permitindo que bolhas internas de ar escapassem livremente pelo flange. Antes da primeira medição, houve um intervalo de tempo de $24 \mathrm{~h}$ para permitir o equilíbrio entre o seepage meter e o sedimento.

Os coletores plásticos foram preenchidos com cerca de 500 a $1000 \mathrm{~mL}$ de água antes de serem acoplados para as medições, o que equivale a cerca de $1 / 3$ do volume total do coletor, não difi- cultando a entrada nem a saída de água. Este procedimento, além de permitir quantificar os fluxos sentido superficial-subterrâneo, é importante para mitigar resultados anômalos causados pela resistência do coletor plástico, que pode dificultar ou promover o fluxo de água devido à sua expansão, conforme descrito por Shaw e Prepas (1989).

Em cada estação de monitoramento foram instaladas triplicatas dos equipamentos, posicionados em forma de transecto perpendicular à linha de costa, resultando em 15 equipamentos instalados. A distância entre os seepage meters variou entre 2 e 5 metros.

Entre os dias 02/08/2017 e 12/12/2017 foram feitas 15 cam- 
panhas de campo para realizar as medições das descargas subterrâneas nos seepage meters. As medições em todas as estações amostrais foram realizadas no mesmo período do dia, de modo que todos os equipamentos estivessem expostos às mesmas condições climáticas e gradientes hidráulicos. Inicialmente, foram pesados e acoplados os coletores aos seepages meters em todas as estações. Dado o tempo necessário, era feita a retirada dos coletores e medição de volume em cada estação de monitoramento. No total, foram obtidas 169 amostras validadas.

\subsection{Estimativa das Descargas de Águas Subterrâneas}

A vazão e a direção do fluxo entre as águas superficiais e subterrâneas são medidas pela variação do volume de água coletada durante determinado período. 0 período de amostragem necessário varia de acordo com a magnitude das descargas e volume do coletor. Ao final da medição, o coletor não deve estar completamente cheio ou vazio, pois pode mascarar os resultados. Os períodos de medição deste estudo variaram entre 1 e $4 \mathrm{~h}$.

A medição do volume nos coletores foi realizada no início e no fim do período de amostragem, utilizando balança de precisão ou proveta de $1000 \mathrm{~mL}$ graduada em $10 \mathrm{~mL}$. As DAS são calculadas pela Equação 1.

$$
D A S=\frac{V_{\text {final }}-V_{\text {inicial }}}{\Delta T \times \mathrm{A}} \times 0,144
$$

Onde DAS: descargas de águas subterrâneas (cm/dia); V: Volume inicial e final amostrado ( $\mathrm{mL}) ; \Delta \mathrm{T}$ : Período de amostragem (min); A: Área do seepage meter $\left(\mathrm{m}^{2}\right)$. A Equação 1 ainda inclui o fator de conversão de unidades $(0,144)$. Quando o fluxo ocorre no sentido superficial-subterrâneo, o volume final é menor que o inicial, convencionando a classificação de "fluxo negativo". Enquanto que, para o "fluxo positivo", considerou-se o movimento do manancial subterrâneo para compartimento superficial, pois o volume final no coletor é maior que o volume inicial.

\subsection{Monitoramento do nível d'água na laguna}

Para acompanhar a dinâmica do nível d'água da laguna, em fevereiro de 2017 foi instalada uma régua linimétrica para a aferição do nível da laguna nos dias de campanhas de monitoramento. A régua foi instalada na estação PA, nas coordenadas $6945174 \mathrm{~N}$, 750734 L, 22 J. Foi fixada uma estrutura de madeira de 2 metros no sedimento da laguna e escorada na estrutura de um extinto trapiche. Foi instalado um batente, de modo que a régua metálica pudesse ser alocada no momento da medição e retirada em seguida. As leituras de nível na régua são medições relativas, não estando amarradas a um datum referencial de nível.

\section{RESULTADOS E DISCUSSÃO}

\subsection{0 uso do método Seepage Meter}

A partir do método de quantificação de DAS seepage meter, foi possível realizar um diagnóstico inicial e quantificação dos padrões de interação entre as águas superficiais e subterrâneas em diferentes locais da área de estudo, com metodologia acessível e de baixo custo financeiro.
Embora a metodologia aparente ser simples, na prática há diversas dificuldades na aplicação dos seepage meters. Erros de medição foram identificados e que podem ocorrer devido ao design e operação dos equipamentos, dentre eles:

- $\quad$ processos de advecção intersticial (efeito Bernoulli) causados pelo movimento da água como ondas e correntes, podendo influenciar em resultados anômalos, conforme verificado por Cable et al. (2006);

- os fluxos medidos podem não estar relacionados totalmente a fluxos subterrâneos, pois também podem provir de fluxos subsuperficiais como recirculação de águas superficiais. Para isso, é necessária a correta instalação dos tambores, de modo que atinjam profundidade adequada.

- a conexão entre o coletor plástico e o tubo conector é uma possível grande fonte de erro e de perda de amostras. A conexão deve ser feita de maneira a vedar a entrada/saída de água para o ambiente e não impossibilitar a passagem de água do saco coletor para o tambor. Diversos métodos de vedação foram testados, como fita de auto fusão, abraçadeiras plásticas e tubo de látex. 0 método com melhor aproveitamento foi o de tiras de câmara de pneu automotiva;

- perda de seepage meters: ao todo foram perdidos 6 tambores, possivelmente devido ao constante movimento do leito arenoso, fazendo com que os seepage meters se soltassem. Também não é descartada a ação de roubo e vandalismo;

- micro furos nos sacos coletores: houve dificuldade em garantir a integridade dos coletores plásticos, pois estes devem ser finos o suficiente para não interferirem no fluxo natural das águas, porém, no manuseio e transporte, alguns coletores se danificavam e furavam, interferindo nos resultados. Também há a possibilidade de que a degradação dos coletores tenha sido causada pela carcinofauna e ictiofauna associada;

- dimensionamento dos coletores: em algumas medições nas estações PA e LIC, o volume inicial de água dos coletores foi insuficiente para a descarga local (fluxo negativo), ficando totalmente vazios.

Em virtude das dificuldades de aplicação, é necessária uma análise crítica em relação aos resultados apresentados. A variabilidade dos dados coletados e a apresentação de descargas anômalas podem estar intrinsecamente relacionadas à metodologia. Para contornar possiveis fontes de erros, a aplicação em triplicatas em cada estação amostral foi determinante.

É recomendável que a metodologia seja utilizada em conjunto a outros métodos de quantificação indiretos, como mini piezômetros e traçadores. Este trabalho está inserido em uma ampla pesquisa de fluxos subterrâneos na área de estudo, cujos trabalhos em andamento, utilizando mini piezômetros e piezomanômetros vem confirmando os mesmos padrões de interação Asub-Asup.

Assim, mesmo com as diversas dificuldades e significantes limitações, o seepage meter apresentou resultados importantes para o primeiro diagnóstico das interações Asub-Asup na área de estudo, possibilitando a identificação de padrões por meio de medições diretas. 


\subsection{Descarga de Águas Subterrâneas na Lagoa da Conceição}

A partir da amostragem realizada com o seepage meter, três padrões distintos de interação entre Asub-Asup na laguna foram identificados.

Na estação LIC, o fluxo é majoritariamente no sentido superficial- subterrâneo (fluxo negativo), ocorrendo infiltrações de ordem de grandeza até 3 vezes superior às outras estações, chegando a valores de até $-16 \mathrm{~cm} / \mathrm{d}$ (Figura $5 \mathrm{~A}$ ). A mediana da triplicata foi de $-5,51 \mathrm{~cm} / \mathrm{d}$ (Figura 5B). 0 padrão de fluxo superficial-subterrâneo está possivelmente relacionado a poços de captação de água subterrânea próximos à estação. Não foi possível obter informações quanto à vazão de captação e nível dinâmico desses poços.

Figura 5 - Diagrama de caixa das descargas de interação Asub-Asup na Lagoa da Conceição por (a) seepage meter e (b) agrupados por estação
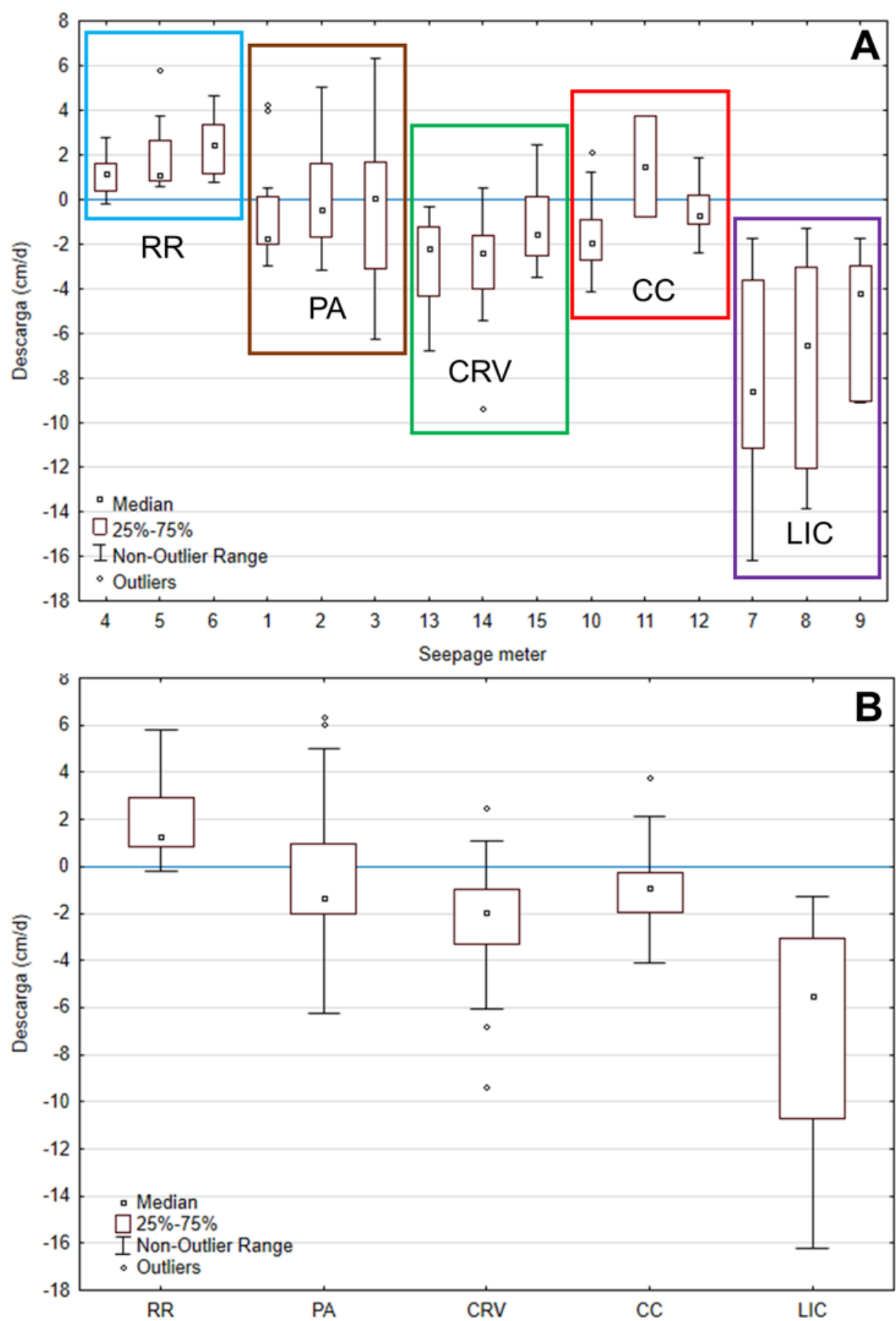

Na estação RR, o fluxo ocorreu no sentido subterrâneo-superficial (fluxo positivo) durante todo o período amostrado. De modo geral, as descargas variaram entre 0,5 a $6,0 \mathrm{~cm} / \mathrm{d}$, com descarga média de $1,30 \mathrm{~cm} / \mathrm{d}$ (Figura 5B). 0 fluxo positivo está associado às descargas do aquífero do campo de dunas próximo à estação. 0 aquí- fero recebe recarga por infiltração através da lagoa de evapoinfiltração da Estação de Tratamento de Esgotos da Companhia Catarinense de Águas e Saneamento (CASAN), localizada a aproximadamente $300 \mathrm{~m}$ da estação RR, medidos em perpendicular à linha de costa. As observações na estação se mostraram estáveis ao 
longo do monitoramento e não apresentaram relação com as variações do nível no corpo lagunar.

Nas estações do corpo central da laguna (CC, CRV e PA) os dados levantados apresentaram uma maior variabilidade temporal e sugerem um padrão espacial do fluxo (Figura 6). Na estação CC, na base do maciço cristalino, as descargas médias resultaram sempre em valores negativos (entre $-0,4$ e $-1,7 \mathrm{~cm} / \mathrm{d}$ ). Os valores negativos se intensificam na estação CRV, localizada no cordão lagunar-praial, onde apresentou valores médios entre -1,6 e -5,3 $\mathrm{cm} / \mathrm{d}$. As infiltrações na estação PA seguem um padrão semelhante as da estação $\mathrm{CC}$, com média de $-1,0 \mathrm{~cm} / \mathrm{d}$, porém algumas medições apresentaram descargas positivas. A diferença entre a estação PA e CC está relacionada a sub-bacia adjacente da esta- ção PA possuir menor declividade e ocorrer a presença de areias quartzolíticas, além do argissolo, possibilitando maior infiltração para o aquífero.

A Figura 6 apresenta as medições realizadas nas estações do corpo central, além da média das DAS entre as estações (DASm) e nível da laguna. A DASm do corpo central ao longo do período monitorado pode estar relacionada com a variação do nível da laguna, indicando que a dinâmica nesta região está possivelmente associada a escala temporal de eventos meteorológicos que afetam diretamente as relações entre nível interno da laguna e nível oceânico. A presença de um gradiente espacial entre as três estações implica em fluxos subterrâneos da vertente rochosa para o lado oceânico da laguna.

Figura 6 - DAS nas estações do corpo central da laguna. A linha contínua indica as descargas de águas subterrâneas médias (DASm) nas três estações. A linha pontilhada indica o nível relativo da laguna

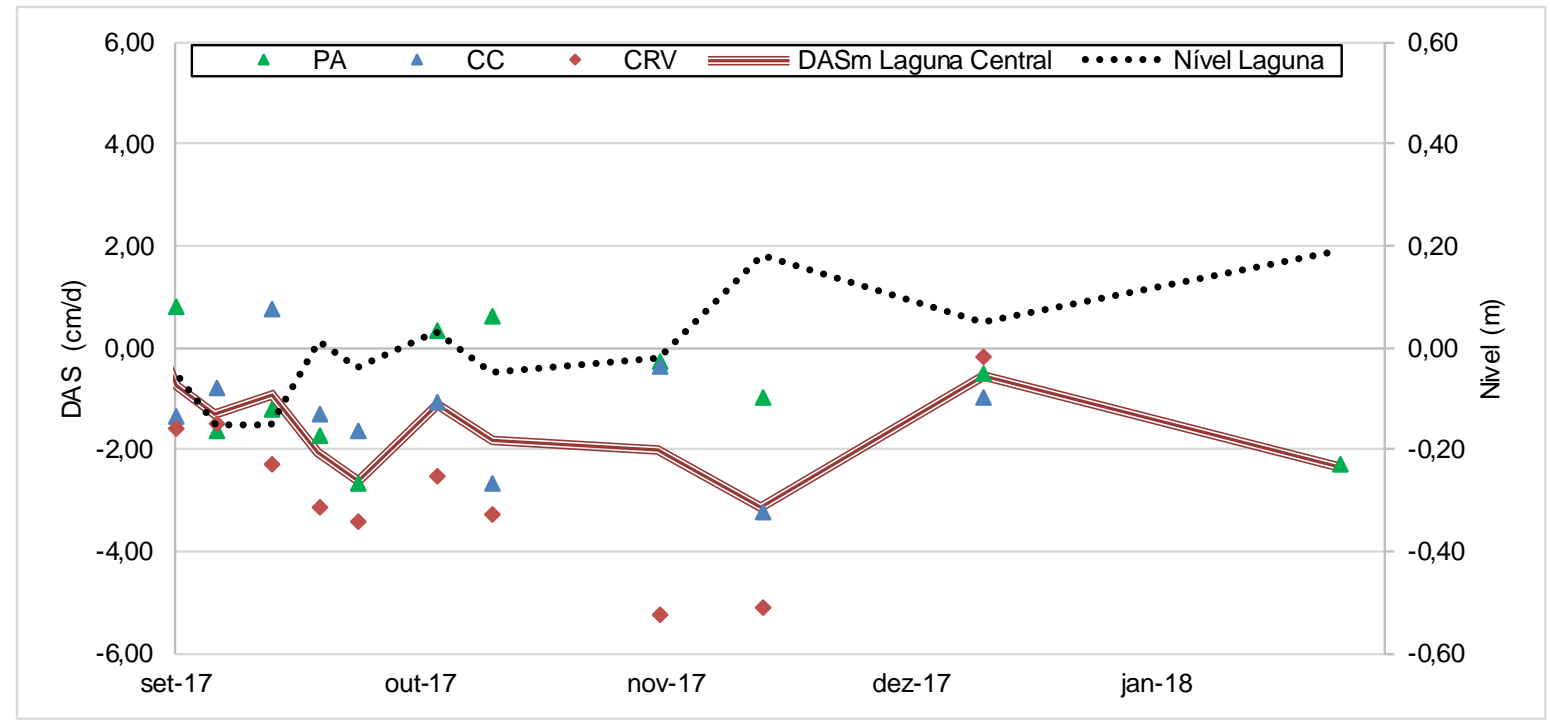

\subsection{Modelos Conceituais}

A partir dos resultados obtidos, onde foram identificados diferentes padrões de interação Asub-Asup no local, foram idealizados modelos conceituais das interações nas diferentes regiões da laguna; cabe ressaltar que este é o primeiro estudo com levantamento de dados na área e que os padrões levantados ainda estão em estágio inicial de investigação.

Como mencionado previamente, os padrões distintos observados relacionaram-se às diferentes características das regiões da laguna. Na estação LIC, o fluxo ocorre no sentido superficialsubterrâneo. Este regime é supostamente influenciado por poços de captação para abastecimento. A Figura 7 representa o com- portamento do fluxo local nessa estação. As setas vermelhas indicam o fluxo negativo em relação à laguna (infiltração), que nesta situação abastece o manancial subterrâneo, que, por sua vez, abastece o poço de captação. As setas azuis indicam o sentido do fluxo subterrâneo e o escoamento superficial através de pequenos córregos.

Opostamente, na estação RR ocorre DAS no sentido subterrâneosuperficial devido ao gradiente hidráulico do aquífero adjacente (setas azuis, Figura 8). No local, ocorre uma zona de recarga artificial do aquífero por infiltração através da LEI (setas vermeIhas, Figura 8), localizada cerca de $500 \mathrm{~m}$ da estação amostral. A LEl possui volume de aproximadamente $60.000 \mathrm{~m}^{3}$, com desnível de aproximadamente $+8,0 \mathrm{~m}$ em relação à laguna. 
Figura 7 - Padrão do fluxo de águas subterrâneas na estação LIC. As setas vermelhas indicam o fluxo negativo em relação à laguna (infiltração) As setas azuis indicam o sentido do fluxo subterrâneo e o escoamento superficial através de pequenos córregos. As linhas tracejadas azuis indicam a superfície do nível d'água (N.A.)

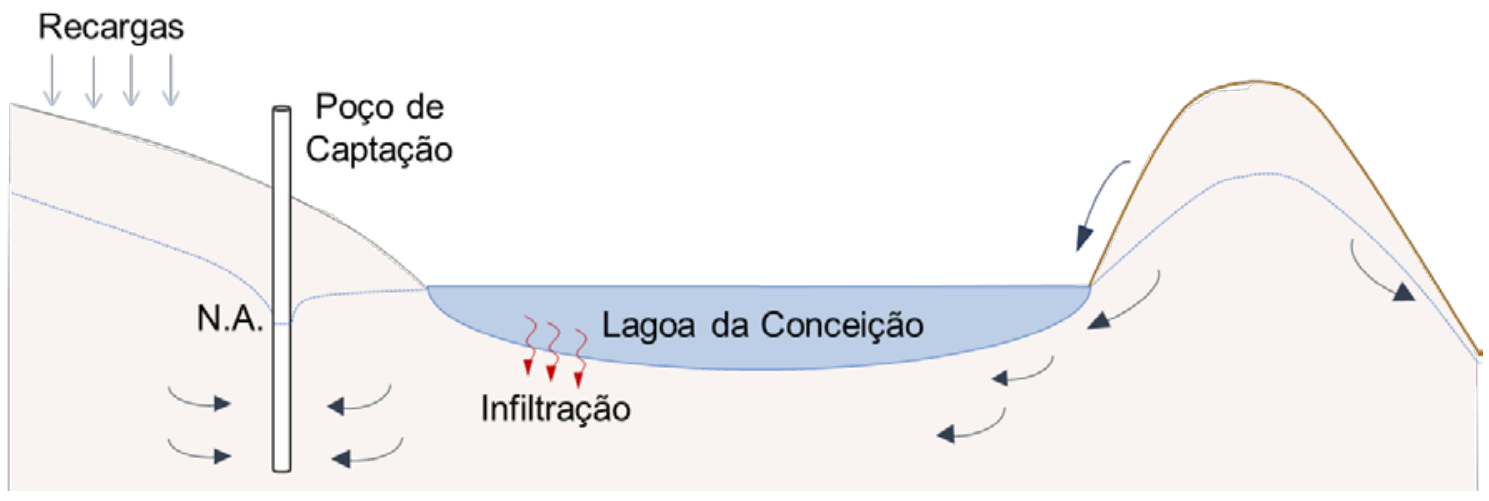

Figura 8 - Influência da lagoa de evapoinfiltração no fluxo de água subterrânea regional da Lagoa da Conceição na estação RR. Setas azuis indicam o fluxo do aquífero para a laguna. Setas vermelhas indicam a infiltração na LEI

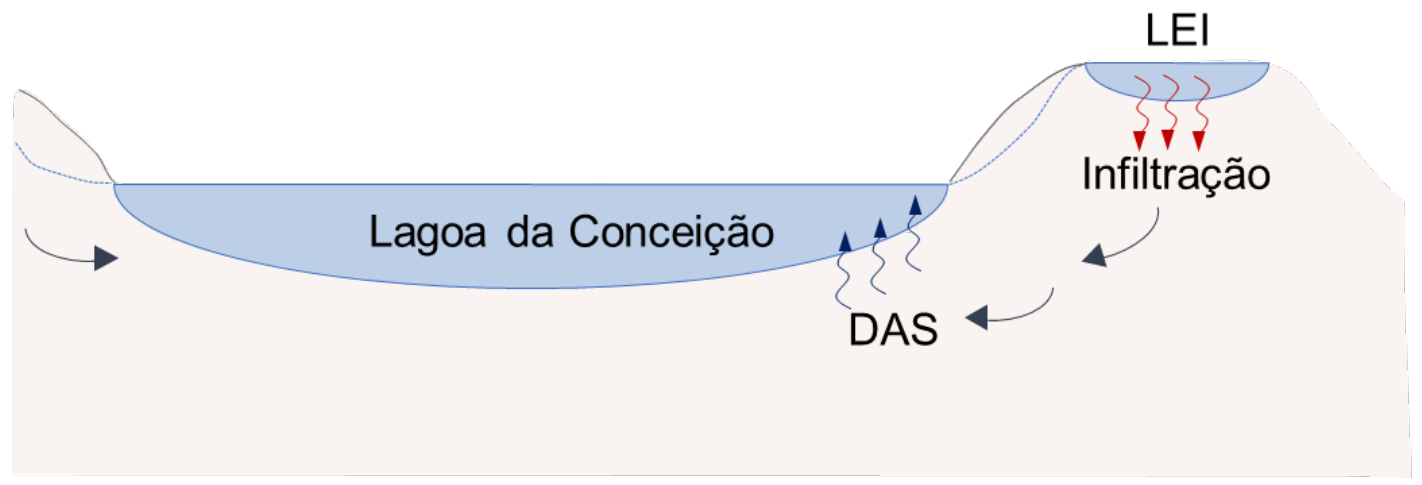

A Figura 9 apresenta o modelo conceitual para o corpo central da laguna (estações CC, CRV, PA). As interações Asub-Asup nesta localidade estão relacionadas a diversas forçantes como a heterogeneidade do aquífero fraturado na margem oeste, da condutividade hidráulica do sedimento, das características da bacia hidrográfica (vegetação, declividade, tipo de solo) e das diferenças de nivel entre a laguna e o oceano. Em relação à forçante variação de nível, no momento em que o nível oceânico se encontra abaixo do nível da laguna, ocorre o fluxo no sentido laguna-aquífero-oceano (setas vermelhas, Figura 9), promovendo a infiltração da água na laguna. Todavia, quando o nível oceânico está acima do nível da laguna, ocorre o movimento oposto, em que o gradiente hidráulico formado entre o nível do oceano e a laguna promove fluxo no sentido oceano-aquífero-laguna, configurando um processo de cunha salina (setas azuis, Figura 9).

Figura 9 - Modelo conceitual das estações do corpo central da laguna (CC, CRV, PA). Comportamento do fluxo subterrâneo (setas vermelhas) com o nível oceânico abaixo do nível da laguna (linha vermelha). As setas azuis indicam o sentido do fluxo quando o nível oceânico se encontra acima do nível da laguna (linha azul). A linha equipotencial preta indica a cunha de intrusão salina e setas pretas o padrão de recirculação

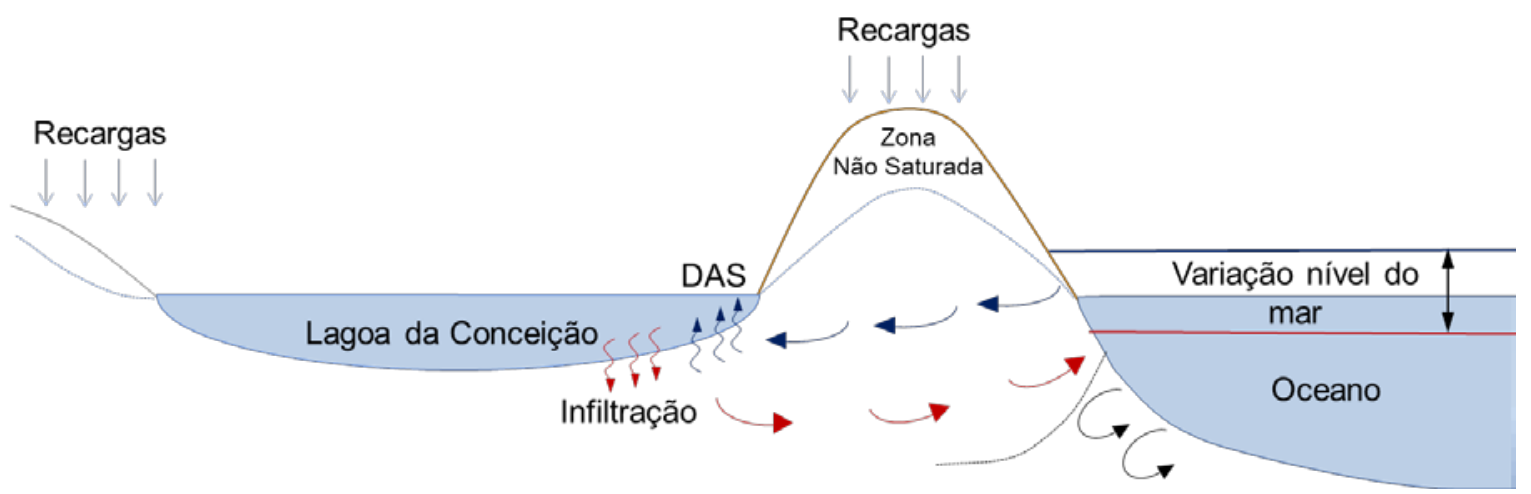

A principal forçante das variações no nível do oceano e da laguna na região é o regime de ventos atuantes. Os ventos do quadrante sul causam o fenômeno de empilhamento, elevando o nível do mar, já os ventos do quadrante norte/nordeste causam o efeito contrário (ANDRADE, 2001; ODRESKI, 2012; OLIVEIRA, 2016). Para o nível da laguna, devido à sinuosidade e comprimento do Canal da Barra da Lagoa, há um efeito de retardamento e filtragem das oscilações do nível oceânico (ANDRADE, 2001; BIER, 
2018; GODOY, 2009), ocasionando o gradiente hidráulico entre os dois sistemas.

Há que se ressaltar as grandes incertezas nesses comportamentos devido a heterogeneidade inerente do sistema no espaço e no tempo, conforme observaram Niencheski et al. (2007) para o sistema Lagoa dos Patos-Mirim. O presente trabalho também não contemplou a análise de variação do nível oceânico, bem como sua diferença em relação ao nível lagunar. Entretanto, este estudo apresentou o primeiro modelo conceitual das interações AsubAsup na Lagoa da Conceição, que deverá ser consolidado com novas abordagens.

\section{CONCLUSÃO}

O presente estudo realizou a primeira investigação da interação Asub-Asup na Lagoa da Conceição a partir de metodologia de quantificação direta e pontual. 0 trabalho apresentou resultados importantes para o primeiro diagnóstico das interações Asub-Asup na área de estudo. Todavia, a aplicação dos seepage meters em campo se mostrou mais dificultosa do que o esperado, onde erros de medição foram identificados.

Observaram-se diferentes padrões nas DAS no corpo hídrico da Lagoa da Conceição. Presume-se que estes estejam intrinsecamente relacionados ao uso dos recursos hídricos localmente. Sugere-se também que o regime de DAS está relacionado ao gradiente hidráulico promovido em função das eventuais diferenças de nível entre a laguna e o oceano.

Diante dos resultados observados, com exceção da estação RR, os fluxos na interface Asub-Asup da Lagoa da Conceição fluem no sentido laguna-aquífero, com infiltrações médias entre -0,9 a -5,5 $\mathrm{cm} / \mathrm{d}$. Na estação RR, o fluxo ocorre no sentido subterrâneosuperficial (fluxo positivo), com descargas variando entre 0,5 a 6,0 $\mathrm{cm} / \mathrm{d}$, e descarga média de $1,30 \mathrm{~cm} / \mathrm{d}$ durante o período monitorado.

Recomendam-se estudos confirmatórios com a utilização de métodos integradores, como traçadores geoquímicos, assim como estudos relacionados à qualidade da água e à influência dos aportes subterrâneos de nutrientes nos ciclos biogeoquímicos.

\section{AGRADECIMENTOS}

O presente trabalho contou com apoio do CNPq, Conselho Nacional de Desenvolvimento Científico e Tecnológico - Brasil (134019/2016-4).

\section{REFERÊNCIAS}

ANDRADE, C. F. F. et al. Fluxos de nutrientes associados às descargas de água subterrânea para a Lagoa Mangueira (Rio Grande do Sul, Brasil). Quimica Nova, v. 35, n. 1, p. 5-10, 2012.

ANDRADE, R. C. Estudo da circulação hidrodinâmica da Lagoa da Conceição-SC. Universidade Federal do Rio de Janeiro, 2001.

ARANTES, E. J. Emprego de infiltrômetros na caracterização da interação entre rio e aquifero. Universidade de São Paulo, 2003.

ATTISANO, K. K. et al. Evidences of continental groundwater inputs to the shelf zone in Albardão, RS, Brazil. Brazilian Journal of Oceanography, v. 56 n. 3, p. 189-200, 2008.
ATTISANO, K. K. Aporte subterrâneo: uma fonte complementar de nutrientes para a Costa Sul do Brasil e plataforma adjacente. p. 204, 2012.

BIER, F. B. Caracterização morfométrica e hidrológica da bacia hidrográfica da Lagoa da Conceição, Florianópolis - SC. Universidade Federal de Santa Catarina, 2013.

BIER, F. B. Aspectos hidrodinâmicos e sedimentológicos em um canal de maré: estudo de caso do Canal da Barra da Lagoa, Florianópolis - SC. Universidade Federal de Santa Catarina, 2018.

BURNETT, W. C. et al. Groundwater and pore water inpunts to the coastal zone. Biogeochemistry, v. 66, p. 3-33, 2003.

BURNETT, W. C. et al. Quantifying submarine groundwater discharge in the coastal zone via multiple methods. Science of the Total Environment, v. 367, n. 2-3, p. 498-543, 2006.

CABLE, J. E.; MARTIN, J. B.; JAEGER, J. Exonerating Bernoulli? On evaluating the physical and biological processes affecting marine seepage meter measurements. Limnology and Oceanography: methods, v. 4, p. 172-183, 2006.

CURY, V. E. S. et al. Space time evolution of the trophic state of a subtropical lagoon: Lagoa da Conceição , Florianópolis Island of Santa Catarina, Brazil. RBRH - Revista Brasileira de Recursos Hídricos, v. 22, n. 10, p. 17, 2017.

DEBNATH, P.; MUKHERJEE, A. Quantification of tidally-influenced seasonal groundwater discharge to the Bay of Bengal by seepage meter study. Journal of Hydrology, v. 537, p. 106-116, 2016.

FONSECA, A. Efeito da drenagem urbana nas características físico-químicas e biológicas da água superficial na Lagoa da Conceição (Florianópolis, SC, Brasil). Revista Biotemas, v. 19, n. 2, p. 7-16, 2006.

GODOY, F. B. Modelagem hidrológico-hidrodinâmica da Lagoa da Conceição - SC. Universidade Federal de Santa Catarina, 2009.

JI, T. et al. Nutrient inputs to a Lagoon through submarine groundwater discharge: The case of Laoye Lagoon, Hainan, China. Journal of Marine Systems, v. 111-112, p. 253-262, 2013.

LEE, D. R. A device for measuring seepage flux in lakes and estuaries. Limnology and Oceanography, v. 22, n. 1, p. 140-147, 1977.

LEOTE, C.; IBÁNHEZ, J. S.; ROCHA, C. Submarine groundwater discharge as a nitrogen source to the Ria Formosa studied with seepage meters. Biogeochemistry, v. 88, n. 2, p. 185-194, 2008.

LEWANDOWSKI, J. et al. From submarine to lacustrine groundwater discharge. IAHS-AISH Proceedings and Reports, v. 365, n. July 2013, p. $72-78,2014$.

MARTIN, J. B. et al. Magnitudes of submarine groundwater discharge from marine and terrestrial sources: Indian River Lagoon, Florida. Water Resources Research, v. 43, n. 5, p. 1-15, 2007.

MOORE, W. S. The Effect of Submarine Groundwater Discharge on the Ocean. Annual Review of Marine Science, v. 2, n. 1, p. 59-88, 2010.

MUEHE, D.; CARUSO JR., F. Batimetria e algumas considerações sobre a evolução geológica da Lagoa da Conceição - Ilha de Santa Catarina. Geosul, n. 7, p. 32-45, 1989.

MURDOCH, L. C.; KELLY, S. E. Factors affecting the performance of conventional seepage meters. Water Resources Research, v. 39, n. 6, p. 1-10, 2003.

NIENCHESKI, L. F. H. et al. Submarine groundwater discharge of nutrients to the ocean along a coastal lagoon barrier, Southern Brazil. Marine Chemistry, v. 106, n. 3-4, p. 546-561, 2007.

ODRESKI, L. F. R. Influêcia hidrológica nos processos hidrodinâmicos da Lagoa da Conceição - Florianópolis - SC. Universidade Federal de Santa Catarina, 2012.

OLIVEIRA, A. DE O. Susceptibilidade e vulnerabilidade de sistemas costeiros abrigados à oscilação positiva do nível do mar: Estudo de caso 
Lagoa da Conceição - Florianópolis/SC. 2016.

PEREIRA, M. L. M. Estudo da Dinâmica das Águas do Canal da Barra da Lagoa - Florianópolis, SC. Universidade Federal de Santa Catarina, 2004.

PORTO FILHO, É. Sedimentometria e algumas considerações sobre a biogeoquímica dos sedimentos de fundo da Lagoa da Conceição, Ilha de Santa Catarina. Universidade Federal de Santa Catarina, 1993.

ROCHA, C. M.; ANDRADE, C. F. F.; NIENCHESKI, L. F. H. Descarga de água subterrânea para as lagoas costeiras do Litoral Norte do Rio Grande do Sul, Brasil. Brazilian Journal of Aquatic Science and Technology, v. 19, n. 2, p. 76-84, 2015.

ROCHA, C. B. M. DA. Implementação e uso do modelo Mike 21 ná analise de cenários hidrodinâmicos na Lagoa da Conceição - SC. [s.I.] Universidade Federal de Santa Catarina, 2007.

ROSENBERRY, D. O.; MENHEER, M. A. A System for Calibrating Seepage Meters Used to Measure Flow Between Ground Water and Surface Water. Scientific Investigations Report 2005-5053, 2006.

SANTOS, I. R. et al. Tracing anthropogenically driven groundwater discharge into a coastal lagoon from southern Brazil. Journal of Hydrology, v. 353, n.
$3-4$, p. $275-293,2008$

SHAW, R. D.; PREPAS, E. E. Anomalous, short-term influx of water into seepage meters. Limnology and Oceanography, v. 34, n. 7, p. 1343-1351, 1989.

SIERRA DE LEDO, B.; SORIANO-SIERRA, E. O Ecossistema da Lagoa da Conceição. 1. ed. Florianópolis: NEMAR - CCB - UFSC, 1999.

SILVA, V. E. C. Análise espaço temporal do estado trófico de uma laguna costeira subtropica: Lagoa da Conceição, Florianópolis, Santa Catarina. [s.I.] Universidade Federal de Santa Catarina, 2016.

SOLDER, J. E. et al. A Tube Seepage Meter for In Situ Measurement of Seepage Rate and Groundwater Sampling. Groundwater, v. 54, n. 4, p. 588-595, 2016.

SOPHOCLEOUS, M. Interactions between groundwater and surface water: The state of the science. Hydrogeology Journal, v. 10, n. 1, p. 52-67, 2002.

WINTER, T. C. et al. Ground water and surface water: a single resource. U.S Geological Survey Circular 1139, p. 79, 1998. 